\title{
Wigner Distribution Moments Measured as Intensity Moments in Separable First-Order Optical Systems
}

\author{
Martin J. Bastiaans \\ Faculteit Elektrotechniek, Technische Universiteit Eindhoven, Postbus 513, 5600 MB Eindhoven, The Netherlands \\ Email:m.j.bastiaans@tue.nl \\ Tatiana Alieva \\ Facultad de Ciencias Físicas, Universidad Complutense de Madrid, Ciudad Universitaria s/n, Madrid 28040, Spain \\ Email: talieva@fis.ucm.es
}

Received 31 March 2004; Revised 19 August 2004

It is shown how all global Wigner distribution moments of arbitrary order can be measured as intensity moments in the output plane of an appropriate number of separable first-order optical systems (generally anamorphic ones). The minimum number of such systems that are needed for the determination of these moments is derived.

Keywords and phrases: Wigner distribution moments, beam characterization, first-order optical systems.

\section{INTRODUCTION}

After the introduction of the Wigner distribution (WD) [1] for the description of coherent and partially coherent optical fields [2], it became an important tool for optical signal/image analysis and beam characterization $[3,4,5]$. The WD completely describes the complex amplitude of a coherent optical field (up to a constant phase factor) or the mutual coherence function of a partially coherent field. As the WD of a two-dimensional optical field is a function of four variables, it is difficult to analyze. Therefore, the optical field is often represented not by the WD itself, but by its global moments. Beam characterization based on the second-order moments of the WD thus became the basis of an International Organization for Standardization standard [6].

Some of the WD moments can directly be determined from measurements of the intensity distributions in the image plane or the Fourier plane, but most of the moments cannot be determined in such an easy way. In order to calculate such moments, additional information is required. Since first-order optical systems [7] —also called $A B C D$ systemsproduce affine transformations of the WD in phase space, the intensity distributions measured at the output of such systems can provide such additional information. The application of $A B C D$ systems for the measurements of the second-

This is an open access article distributed under the Creative Commons Attribution License, which permits unrestricted use, distribution, and reproduction in any medium, provided the original work is properly cited. order WD moments has been reported in several publications $[8,9,10,11,12,13]$.

It is the aim of this paper to show how all global WD moments can be measured as intensity moments only. We show that not only the second-order moments, but also all other moments of the four-dimensional WD can be obtained from measurements of only intensity distributions in an appropriate number of (generally anamorphic) separable first-order optical systems.

\section{WIGNER DISTRIBUTION}

Let partially coherent light be described by a temporally stationary stochastic process $f(x, y ; t)$; as far as the time dependence is concerned, the ensemble average of the product $f\left(x_{1}, y_{1} ; t_{1}\right) f^{*}\left(x_{2}, y_{2} ; t_{2}\right)$, where the asterisk denotes complex conjugation, is then only a function of the time difference $t_{1}-t_{2}:$

$$
\mathrm{E}\left\{f\left(x_{1}, y_{1} ; t_{1}\right) f^{*}\left(x_{2}, y_{2} ; t_{2}\right)\right\}=\gamma\left(x_{1}, x_{2} ; y_{1}, y_{2} ; t_{1}-t_{2}\right) .
$$

The function $\gamma\left(x_{1}, x_{2} ; y_{1}, y_{2} ; \tau\right)$ is known as the mutual coherence function $[14,15,16,17]$ of the stochastic process $f(x, y ; t)$. The mutual power spectrum $[16,17]$ or crossspectral density function [18] $\Gamma\left(x_{1}, x_{2} ; y_{1}, y_{2} ; \omega\right)$ is defined as the temporal Fourier transform of the mutual coherence function:

$$
\begin{aligned}
& \Gamma\left(x_{1}, x_{2} ; y_{1}, y_{2} ; \omega\right) \\
& \quad=\int_{-\infty}^{\infty} \gamma\left(x_{1}, x_{2} ; y_{1}, y_{2} ; \tau\right) \exp (j \omega \tau) d \tau .
\end{aligned}
$$


For $x_{1}=x_{2}=x, y_{1}=y_{2}=y$, the cross-spectral density function reduces to the (auto) power spectrum $\Gamma(x, x ; y, y ; \omega)$, which represents the intensity distribution of the light for the temporal frequency $\omega$. Since in the present discussion the explicit temporal-frequency dependence is of no importance, we will, for the sake of convenience, omit the temporal-frequency variable $\omega$ from the formulas in the remainder of the paper.

The Wigner distribution of partially coherent light is defined in terms of the cross-spectral density function by

$$
\begin{gathered}
W(x, u ; y, v) \\
=\int_{-\infty}^{\infty} \int_{-\infty}^{\infty} \Gamma\left(x+\frac{1}{2} x^{\prime}, x-\frac{1}{2} x^{\prime} ; y+\frac{1}{2} y^{\prime}, y-\frac{1}{2} y^{\prime}\right) \\
\times \exp \left[-j 2 \pi\left(u x^{\prime}+v y^{\prime}\right)\right] d x^{\prime} d y^{\prime}
\end{gathered}
$$

A distribution function according to definition (3) was first introduced in optics by Walther [19, 20], who called it the generalized radiance. The $\mathrm{WD} W(x, u ; y, v)$ represents partially coherent light in a combined space/spatial-frequency domain, the so-called phase space, where $u$ is the spatialfrequency variable associated to the space variable $x$, and $v$ the spatial-frequency variable associated to the space variable $y$.

In this paper we consider the normalized moments of the $\mathrm{WD}$, where the normalization is with respect to the total energy $E$ of the signal:

$$
\begin{aligned}
E & =\int_{-\infty}^{\infty} \int_{-\infty}^{\infty} \int_{-\infty}^{\infty} \int_{-\infty}^{\infty} W(x, u ; y, v) d x d u d y d v \\
& =\int_{-\infty}^{\infty} \int_{-\infty}^{\infty} \Gamma(x, x ; y, y) d x d y .
\end{aligned}
$$

These normalized moments $\mu_{\text {pqrs }}$ of the WD are thus defined by

$$
\begin{aligned}
\mu_{p q r s} E & =\int_{-\infty}^{\infty} \int_{-\infty}^{\infty} \int_{-\infty}^{\infty} \int_{-\infty}^{\infty} W(x, u ; y, v) x^{p} u^{q} y^{r} v^{s} d x d u d y d v \quad(p, q, r, s \geq 0) \\
& =\left.\frac{1}{(4 \pi j)^{q+s}} \int_{-\infty}^{\infty} \int_{-\infty}^{\infty} x^{p} y^{r}\left(\frac{\partial}{\partial x_{1}}-\frac{\partial}{\partial x_{2}}\right)^{q}\left(\frac{\partial}{\partial y_{1}}-\frac{\partial}{\partial y_{2}}\right)^{s} \Gamma\left(x_{1}, x_{2} ; y_{1}, y_{2}\right)\right|_{x_{1}=x_{2}=x, y_{1}=y_{2}=y} d x d y .
\end{aligned}
$$

Note that for $q=s=0$ we have intensity moments, which can easily be measured:

$$
\begin{aligned}
& \mu_{p 0 r 0} E \\
& =\int_{-\infty}^{\infty} \int_{-\infty}^{\infty} \int_{-\infty}^{\infty} \int_{-\infty}^{\infty} W(x, u ; y, v) \\
& \quad \times x^{p} y^{r} d x d u d y d v \quad(p, r \geq 0) \\
& =\int_{-\infty}^{\infty} \int_{-\infty}^{\infty} x^{p} y^{r} \Gamma(x, x ; y, y) d x d y .
\end{aligned}
$$

The WD moments $\mu_{\text {pqrs }}$ provide valuable tools for the characterization of optical beams, see, for instance [21]. First-order moments yield the position of the beam $\left(\mu_{1000}\right.$ and $\left.\mu_{0010}\right)$ and its direction $\left(\mu_{0100}\right.$ and $\left.\mu_{0001}\right)$. Second-order moments give information about the spatial width of the beam (the shape $\mu_{2000}$ and $\mu_{0020}$ of the spatial ellipse and its orientation $\mu_{1010}$ ) and the angular width in which the beam is radiating (the shape $\mu_{0200}$ and $\mu_{0002}$ of the spatial-frequency ellipse and its orientation $\left.\mu_{0101}\right)$; moreover, they provide information about its curvature $\left(\mu_{1100}\right.$ and $\left.\mu_{0011}\right)$ and its twist $\left(\mu_{1001}\right.$ and $\left.\mu_{0110}\right)$. Many important beam characterizers, like the overall beam quality [12]

$$
\begin{aligned}
& \left(\mu_{2000} \mu_{0200}-\mu_{1100}^{2}\right)+\left(\mu_{0020} \mu_{0002}-\mu_{0011}^{2}\right) \\
& \quad+2\left(\mu_{1010} \mu_{0101}-\mu_{1001} \mu_{0110}\right)
\end{aligned}
$$

are based on second-order moments. Higher-order moments are used, for instance, to characterize the beam's symmetry and its sharpness [21].

\section{SEPARABLE FIRST-ORDER OPTICAL SYSTEMS}

It is well known that the input-output relationship between the WD $W_{\text {in }}(x, u ; y, v)$ at the input plane and the WD $W_{\text {out }}(x, u ; y, v)$ at the output plane of a separable first-order optical system reads $[3,4,5]$

$$
\begin{aligned}
& W_{\text {out }}(x, u ; y, v) \\
& \quad=W_{\text {in }}\left(d_{x} x-b_{x} u,-c_{x} x+a_{x} u ; d_{y} y-b_{y} v,-c_{y} y+a_{y} v\right) .
\end{aligned}
$$

The coefficients $a_{x}, b_{x}, c_{x}, d_{x}$ and $a_{y}, b_{y}, c_{y}, d_{y}$ are the matrix entries of the symplectic ray transformation matrix [7] that relates the position $x, y$ and direction $u, v$ of an optical ray in the input and the output plane of the first-order optical system:

$$
\left[\begin{array}{l}
x_{\text {out }} \\
y_{\text {out }} \\
u_{\text {out }} \\
v_{\text {out }}
\end{array}\right]=\left[\begin{array}{cccc}
a_{x} & 0 & b_{x} & 0 \\
0 & a_{y} & 0 & b_{y} \\
c_{x} & 0 & d_{x} & 0 \\
0 & c_{y} & 0 & d_{y}
\end{array}\right]\left[\begin{array}{l}
x_{\text {in }} \\
y_{\text {in }} \\
u_{\text {in }} \\
v_{\text {in }}
\end{array}\right]
$$


For separable systems, symplecticity simply reads $a_{x} d_{x}-$ $b_{x} c_{x}=1$ and $a_{y} d_{y}-b_{y} c_{y}=1$. Note that in a first-order optical system, with such a symplectic ray transformation matrix, the total energy $E$, see (4), is invariant.

As examples of first-order optical systems we mention the following in particular:

(i) a section of free space in the paraxial approximation, or "parabolic" system [22] (with $a=d=1, c=0$, and $b$ proportional to the propagation distance $z$ ),

(ii) a fractional Fourier transform system [23], or "elliptic" system [22] (with $a=d=\cos \alpha$ and $b=-c=\sin \alpha$ ),

(iii) a "hyperbolic" system [22] (with $a=d=\cosh \alpha$ and $b=c=\sinh \alpha)$.

These three systems are characterized by one parameter. Other one-parameter first-order optical systems are

(i) a thin lens (with $a=d=1, b=0$, and $c$ inverse proportional to the focal distance),

(ii) an ideal magnifier (with $a=m, d=1 / m, b=c=0$ ).

The latter systems however-like all systems for which the input and output planes are conjugate planes-cannot be used to determine the moments, as we will see later, because they have the property $b \equiv 0$.

The normalized moments $\mu_{\text {pqrs }}^{\text {out }}$ of the output WD $W_{\text {out }}(x, u ; y, v)$ are related to the normalized moments $\mu_{p q r s}^{\text {in }}=\mu_{p q r s}$ of the input WD $W_{\text {in }}(x, u ; y, v)$ as

$$
\begin{aligned}
& \mu_{p q r s}^{\text {out }} E \\
& =\int_{-\infty}^{\infty} \int_{-\infty}^{\infty} \int_{-\infty}^{\infty} \int_{-\infty}^{\infty} W_{\text {out }}(x, u ; y, v) \\
& \times x^{p} u^{q} y^{r} v^{s} d x d u d y d v \\
& =\int_{-\infty}^{\infty} \int_{-\infty}^{\infty} \int_{-\infty}^{\infty} \int_{-\infty}^{\infty} W_{\text {in }}\left(d_{x} x-b_{x} u,-c_{x} x+a_{x} u\right. \\
& \left.d_{y} y-b_{y} v,-c_{y} y+a_{y} v\right) \\
& \quad \times x^{p} u^{q} y^{r} v^{s} d x d u d y d v
\end{aligned}
$$

and for the intensity moments in particular (i.e., $q=s=0$ ) we have

$$
\mu_{p 0 r 0}^{\text {out }}=\sum_{k=0}^{p} \sum_{m=0}^{r}\left(\begin{array}{l}
p \\
k
\end{array}\right)\left(\begin{array}{c}
r \\
m
\end{array}\right) a_{x}^{p-k} b_{x}^{k} a_{y}^{r-m} b_{y}^{m} \mu_{p-k, k, r-m, m} .
$$

The remainder of this paper is based on (11), in which the output intensity moments $\mu_{p 0 r 0}^{\text {out }}$ are expressed in terms of the input moments $\mu_{p q r s}$ and the system parameters $a_{x}, a_{y}$, $b_{x}$, and $b_{y}$. Note that only the parameters $a$ and $b$ enter this equation; the parameters $c$ and $d$ can be chosen freely, as long as the symplecticity condition $a_{x} d_{x}-b_{x} c_{x}=a_{y} d_{y}-b_{y} c_{y}=1$ is satisfied.

\section{RELATIONS BETWEEN INPUT AND OUTPUT MOMENTS}

\subsection{First-order moments}

For the first-order moments, the following two equations are relevant:

$$
\begin{aligned}
& \mu_{1000}^{\text {out }}=a_{x} \mu_{1000}+b_{x} \mu_{0100}, \\
& \mu_{0010}^{\text {out }}=a_{y} \mu_{0010}+b_{y} \mu_{0001},
\end{aligned}
$$

which correspond to (11) with pqrs $=1000$ and pqrs $=$ 0010 , respectively, and the four input moments $\mu_{1000}, \mu_{0100}$, $\mu_{0010}$, and $\mu_{0001}$ can be determined by measuring the intensity moments $\mu_{1000}^{\text {out }}$ and $\mu_{0010}^{\text {out }}$ in the output planes of two systems with different values of $a$ and $b$, see (12) and (13), respectively.

In the case of a fractional Fourier transform system we can choose, for instance, $[24,25]$, the fractional angles $\alpha_{x}=$ $\alpha_{y}=0$ (leading to $a_{x}=a_{y}=1$ and $b_{x}=b_{y}=0$ ) and $\alpha_{x}=\alpha_{y}=\pi / 2$ (leading to $a_{x}=a_{y}=0$ and $b_{x}=b_{y}=1$ ), but any other choice could be made as well, as long as it leads to four independent equations. In the case of free space propagation, we simply choose two different values of the propagation distance $z$, corresponding to two different values of $b_{x}$ and $b_{y}$ (with $a_{x}=a_{y}=1$, of course).

Note that the two first-order optical systems can always be chosen such that they are isotropic, $a_{x}=a_{y}=a_{i}, b_{x}=$ $b_{y}=b_{i}$, and so forth $(i=1,2)$, with identical behavior in the $x$ and the $y$ direction.

\subsection{Second-order moments}

For the $3+4+3=10$ second-order moments, the following equations are relevant:

$$
\begin{aligned}
& \mu_{2000}^{\text {out }}=a_{x}^{2} \mu_{2000}+2 a_{x} b_{x} \mu_{1100}+b_{x}^{2} \mu_{0200}, \\
& \mu_{1010}^{\text {out }}=a_{x} a_{y} \mu_{1010}+a_{x} b_{y} \mu_{1001}+b_{x} a_{y} \mu_{0110}+b_{x} b_{y} \mu_{0101}, \\
& \mu_{0020}^{\text {out }}=a_{y}^{2} \mu_{0020}+2 a_{y} b_{y} \mu_{0011}+b_{y}^{2} \mu_{0002},
\end{aligned}
$$

which equations correspond to (11) with pqrs $=2000$, pqrs $=1010$, and pqrs $=0020$, respectively.

The three input moments $\mu_{2000}, \mu_{1100}$, and $\mu_{0200}$ can be determined by measuring the intensity moment $\mu_{2000}^{\text {out }}$ in the output planes of three systems with different values of $a_{x}$ and $b_{x}$, see (14). Likewise, with the transversal coordinate $x$ replaced by $y$, the three input moments $\mu_{0020}, \mu_{0011}$, and $\mu_{0002}$ can be determined by measuring the intensity moment $\mu_{0020}^{\text {out }}$ 
in the output planes of three systems with different values of $a_{y}$ and $b_{y}$, see (15). Note that we can choose $a_{x}=a_{y}=a_{i}$ and $b_{x}=b_{y}=b_{i}(i=1,2,3)$ for these three systems, in which case we are obviously using isotropic systems.

The other four input moments $\mu_{1010}, \mu_{1001}, \mu_{0110}$, and $\mu_{0101}$ follow from measuring the intensity moment $\mu_{1010}^{\text {out }}$ in the output planes of four different systems, see (15). However, if we would use only isotropic systems, like we could do for (14) and (16), (15) would reduce to

$$
\mu_{1010}^{\text {out }}=a^{2} \mu_{1010}+a b\left(\mu_{1001}+\mu_{0110}\right)+b^{2} \mu_{0101}
$$

and we can only determine the combination $\mu_{1001}+\mu_{0110}$. Hence, while three systems may be isotropic again - and, for instance, be identical to the ones that we used when we were dealing with (14) and (16) — at least one system should be anamorphic.

We conclude that all ten second-order moments can be determined from the knowledge of the output intensities of four first-order optical systems, where one of them has to be anamorphic. In the case of fractional Fourier transform systems we could choose, for instance $[24,25]$, the fractional angles $\alpha_{x}=\alpha_{y}=0$ (leading to $a_{x}=a_{y}=1$ and $b_{x}=b_{y}=0$ ), $\alpha_{x}=\alpha_{y}=\pi / 4$ (leading to $a_{x}=a_{y}=b_{x}=b_{y}=\sqrt{2} / 2$ ), $\alpha_{x}=\alpha_{y}=\pi / 2$ (leading to $a_{x}=a_{y}=0$ and $b_{x}=b_{y}=1$ ), and the anamorphic combination $\alpha_{x}=\pi / 2 \pi$ and $\alpha_{y}=0$ (leading to $a_{x}=b_{y}=0$ and $a_{y}=b_{x}=1$ ). If we decide to determine the moments using free space propagation, we should be aware of the fact that an anamorphic free space system cannot be realized by mere free space, but can only be simulated by using a proper arrangement of cylindrical lenses.

Of course, optical schemes to determine all ten secondorder moments have been described before, see, for instance $[8,9,11,12,13]$, but the way to determine these moments as presented in this paper is based on a general scheme that can also be used for the determination of arbitrary higher-order moments.

\subsection{Higher-order moments}

For higher-order moments we can proceed analogously. For the $4+6+6+4=20$ third-order moments, the following equations are relevant:

$$
\begin{aligned}
\mu_{3000}^{\text {out }}= & a_{x}^{3} \mu_{3000}+3 a_{x}^{2} b_{x} \mu_{2100}+3 a_{x} b_{x}^{2} \mu_{1200}+b_{x}^{3} \mu_{0300}, \\
\mu_{2010}^{\text {out }}= & a_{x}^{2} a_{y} \mu_{2010}+a_{x}^{2} b_{y} \mu_{2001}+2 a_{x} b_{x} a_{y} \mu_{1110} \\
& +2 a_{x} b_{x} b_{y} \mu_{1101}+b_{x}^{2} a_{y} \mu_{0210}+b_{x}^{2} b_{y} \mu_{0201}, \\
\mu_{1020}^{\text {out }}= & a_{x} a_{y}^{2} \mu_{1020}+2 a_{x} a_{y} b_{y} \mu_{1011}+a_{x} b_{y}^{2} \mu_{1002} \\
& +b_{x} a_{y}^{2} \mu_{0120}+2 b_{x} a_{y} b_{y} \mu_{0111}+b_{x} b_{y}^{2} \mu_{0102}, \\
\mu_{0030}^{\text {out }}= & a_{y}^{3} \mu_{0030}+3 a_{y}^{2} b_{y} \mu_{0021}+3 a_{y} b_{y}^{2} \mu_{0012}+b_{y}^{3} \mu_{0003} .
\end{aligned}
$$

Note again that these equations correspond to (11) with pqrs $=3000$, pqrs $=2010$, pqrs $=1020$, and pqrs $=0030$, respectively. The 20 third-order moments can be determined from the knowledge of the output intensities of six first-order optical systems, where two of them have to be anamorphic.
We consider in more detail how the third-order moments could be determined.

(i) The four input moments $\mu_{3000}, \mu_{2100}, \mu_{1200}$, and $\mu_{0300}$ can be determined by measuring the intensity moment $\mu_{3000, i}^{\text {out }}(i=1,2,3,4)$ in the output planes of four systems with different values of $a_{x}$ and $b_{x}$, see (18). Likewise, with the transversal coordinate $x$ replaced by $y$, the four input moments $\mu_{0030}, \mu_{0021}, \mu_{0012}$, and $\mu_{0003}$ can be determined by measuring the intensity moment $\mu_{0030, i}^{\text {out }}(i=1,2,3,4)$ in the output planes of four systems with different values of $a_{y}$ and $b_{y}$, see (21). Note that we can choose $a_{x}=a_{y}=a_{i}$ and $b_{x}=b_{y}=b_{i}$ $(i=1,2,3,4)$ for these four different systems, in which case we are obviously using isotropic systems. This then leads to the set of four equations

$$
\begin{gathered}
a_{i}^{3} \mu_{3000}+3 a_{i}^{2} b_{i} \mu_{2100}+3 a_{i} b_{i}^{2} \mu_{1200}+b_{i}^{3} \mu_{0300} \\
=\mu_{3000, i}^{\text {out }} \quad(i=1,2,3,4)
\end{gathered}
$$

based on (18) and a similar set of four equations

$$
\begin{gathered}
a_{i}^{3} \mu_{0030}+3 a_{i}^{2} b_{i} \mu_{0021}+3 a_{i} b_{i}^{2} \mu_{0012}+b_{i}^{3} \mu_{0003} \\
=\mu_{0030, i}^{\text {out }} \quad(i=1,2,3,4),
\end{gathered}
$$

based on (21). Possible system choices are, for instance, four sections of free space, with $a_{i}=1$ and $b_{i}$ proportional to the four different propagation distances $z_{i}(i=1,2,3,4)$; or four isotropic fractional Fourier transform systems with $a_{i}=\cos \alpha_{i}$ and $b_{i}=\sin \alpha_{i}$, and $\alpha_{i}(i=1,2,3,4)$ four different fractional angles.

(ii) Using the same four isotropic systems as above, the two input moments $\mu_{2010}$ and $\mu_{0201}$, together with the two moment combinations $\mu_{2001}+2 \mu_{1110}$ and $2 \mu_{1101}+\mu_{0210}$, follow from measuring the intensity moment $\mu_{2010, i}^{\text {out }}$ $(i=1,2,3,4)$ in the output planes of these four systems, see (19), while the two input moments $\mu_{1020}$ and $\mu_{0102}$, together with the two moment combinations $2 \mu_{1011}+\mu_{0120}$ and $\mu_{1002}+2 \mu_{0111}$, follow from measuring the intensity moment $\mu_{1020, i}^{\text {out }}(i=1,2,3,4)$, see $(20)$. This leads to the set of four equations

$$
\begin{gathered}
a_{i}^{3} \mu_{2010}+a_{i}^{2} b_{i}\left(\mu_{2001}+2 \mu_{1110}\right)+a_{i} b_{i}^{2}\left(2 \mu_{1101}+\mu_{0210}\right) \\
+b_{i}^{3} \mu_{0201}=\mu_{2010, i}^{\text {out }} \quad(i=1,2,3,4)
\end{gathered}
$$

based on (19) and a similar set of four equations

$$
\begin{gathered}
a_{i}^{3} \mu_{1020}+a_{i}^{2} b_{i}\left(2 \mu_{1011}+\mu_{0120}\right)+a_{i} b_{i}^{2}\left(\mu_{1002}+2 \mu_{0111}\right) \\
+b_{i}^{3} \mu_{0102}=\mu_{1020, i}^{\text {out }} \quad(i=1,2,3,4)
\end{gathered}
$$

based on (20).

(iii) Twelve of the 20 input moments (together with four moment combinations) can thus be determined by using four isotropic systems. To determine the remaining eight moments, we need four more equations based on (19) and (20), for which we have to use two more systems (labeled $i=5$ and $i=6$ ), which should now 
TABLE 1: The number of $n$ th-order moments $N$, and the required number of first-order optical systems to determine these $N$ moments, given as a function of $n$.

\begin{tabular}{ccrrc}
\hline$n$ & Number of $n$ th-order moments & $N$ & $N_{t}$ & $N_{a}$ \\
\hline 0 & 1 & 1 & 1 & 0 \\
1 & $2+2$ & 4 & 2 & 0 \\
2 & $3+4+3$ & 10 & 4 & 1 \\
3 & $4+6+6+4$ & 20 & 6 & 2 \\
4 & $5+8+9+8+5$ & 35 & 9 & 4 \\
5 & $6+10+12+12+10+6$ & 56 & 12 & 6 \\
6 & $7+12+15+16+15+12+7$ & 84 & 16 & 9 \\
$\vdots$ & $\vdots$ & $\vdots$ & $\vdots$ & $\vdots$ \\
\hline
\end{tabular}

be anamorphic. Among the many possibilities, an easy choice would be a system with $a_{x}=b_{y}=0, b_{x} \neq 0$, $a_{y} \neq 0$, leading to

$$
\begin{aligned}
& b_{x}^{2} a_{y} \mu_{0210}=\mu_{2010,5}^{\text {out }}, \\
& b_{x} a_{y}^{2} \mu_{0120}=\mu_{1020,5}^{\text {out }},
\end{aligned}
$$

and a system with $b_{x}=a_{y}=0, a_{x} \neq 0, b_{y} \neq 0$, leading to

$$
\begin{aligned}
& a_{x}^{2} b_{y} \mu_{2001}=\mu_{2010,6}^{\text {out }}, \\
& a_{x} b_{y}^{2} \mu_{1002}=\mu_{1020,6}^{\text {out }} .
\end{aligned}
$$

The former system may be an anamorphic fractional Fourier transform system with fractional angles $\alpha_{x}=$ $\pi / 2$ and $\alpha_{y}=0$ (and hence $a_{x}=b_{y}=0$ and $b_{x}=a_{y}=$ 1 ), while the latter may be an anamorphic fractional Fourier transform system with $\alpha_{x}=0$ and $\alpha_{y}=\pi / 2$ (and hence $b_{x}=a_{y}=0$ and $b_{y}=a_{x}=1$ ).

Altogether we have thus constructed 20 equations for the 20 third-order moments, using a total of six first-order systems: four isotropic systems where we measure the 16 output intensity moments $\mu_{3000, i}, \mu_{0030, i}, \mu_{2010, i}$, and $\mu_{1020, i}(i=$ $1,2,3,4)$, and two anamorphic systems where we measure the four output intensity moments $\mu_{2010, i}^{\text {out }}$ and $\mu_{1020, i}^{\text {out }}(i=$ $5,6)$.

For the $5+8+9+8+5=35$ fourth-order moments, the relevant equations follow from (11) with pqrs $=4000$, pqrs $=3010$, pqrs $=2020$, pqrs $=1030$, and pqrs $=0040$, respectively. The 35 fourth-order moments can be determined from the knowledge of the output intensities of nine first-order optical systems spectra, where four of them have to be anamorphic. Constructing a measuring scheme along the lines described above for the second-order case and the third-order case, is rather straightforward.

To find the number of $n$ th-order moments $N$, and the total number of first-order optical systems $N_{t}$ (with $N_{a}$ the number of anamorphic ones) that we need to determine these $N$ moments, use can be made of the triangle presented in Table 1, which can easily be extended to higher order.

Note that $N$ (the number of $n$ th-order moments) is equal to the sum of the values in the $n$th row of the triangle, $N=$ $(n+1)(n+2)(n+3) / 6$; that $N_{t}$ (the total number of first-order optical systems) is equal to the highest value that appears in the $n$th row of the triangle, $N_{t}=(n+2)^{2} / 4$ for $n=$ even, and $N_{t}=(n+3)(n+1) / 4$ for $n=$ odd; that the number of isotropic systems is $n+1$; and that $N_{a}$ (the number of anamorphic systems) follows from $N_{a}=N_{t}-(n+1)$.

\section{CONCLUSIONS}

We have shown how all global WD moments of arbitrary order can be measured as intensity moments in the output planes of an appropriate number of first-order optical systems (separable, but generally anamorphic ones), and we have derived the minimum number of such systems that are needed for the determination of these moments. The results followed directly from the general relationship (11) that expresses the intensity moments in the output plane of a separable first-order optical system in terms of the moments in the input plane and the system parameters $a_{x}, b_{x}, c_{x}, d_{x}$ and $a_{y}, b_{y}, c_{y}, d_{y}$.

\section{REFERENCES}

[1] E. Wigner, "On the quantum correction for thermodynamic equilibrium," Physical Review, vol. 40, no. 5, pp. 749-759, 1932.

[2] M. J. Bastiaans, "The Wigner distribution function applied to optical signals and systems," Optics Communications, vol. 25, no. 1, pp. 26-30, 1978.

[3] M. J. Bastiaans, "Wigner distribution function and its application to first-order optics," Journal of the Optical Society of America, vol. 69, no. 12, pp. 1710-1716, 1979.

[4] M. J. Bastiaans, "Application of the Wigner distribution function to partially coherent light," Journal of the Optical Society of America $\{A\}$, vol. 3, no. 8, pp. 1227-1238, 1986.

[5] W. Mecklenbräuker and F. Hlawatsch, Eds., The Wigner Distribution - Theory and Applications in Signal Processing, Elsevier, Amsterdam, The Netherlands, 1997.

[6] International Organization for Standardization, Technical Committee/Subcommittee 172/SC9, "Lasers and laser-related equipment-test methods for laser beam parameters-beam widths, divergence angle and beam propagation factor," ISO Doc. 11146: 1999, International Organization for Standardization, Geneva, Switzerland, 1999.

[7] R. K. Luneburg, Mathematical Theory of Optics, University of California Press, Berkeley and Los Angeles, Calif, USA, 1966.

[8] G. Nemes and A. E. Siegman, "Measurement of all ten secondorder moments of an astigmatic beam by the use of rotating simple astigmatic (anamorphic) optics," Journal of the Optical Society of America $\{A\}$, vol. 11, no. 8, pp. 2257-2264, 1994.

[9] B. Eppich, C. Gao, and H. Weber, "Determination of the ten second order intensity moments," Optics \& Laser Technology, vol. 30, no. 5, pp. 337-340, 1998.

[10] R. Simon, N. Mukunda, and E. C. G. Sudarshan, "Partially coherent beams and a generalized ABCD-law," Optics Communications, vol. 65, no. 5, pp. 322-328, 1988.

[11] C. Martínez, F. Encinas-Sanz, J. Serna, P. M. Mejías, and R. Martínez-Herrero, "On the parametric characterization of the transversal spatial structure of laser pulses," Optics Communications, vol. 139, no. 4-6, pp. 299-305, 1997.

[12] J. Serna, R. Martínez-Herrero, and P. M. Mejías, "Parametric characterization of general partially coherent beams propagating through ABCD optical systems," Journal of the Optical Society of America $\{A\}$, vol. 8, no. 7, pp. 1094-1098, 1991. 
[13] J. Serna, F. Encinas-Sanz, and G. Nemes, "Complete spatial characterization of a pulsed doughnut-type beam by use of spherical optics and a cylindrical lens," Journal of the Optical Society of America A, vol. 18, no. 7, pp. 1726-1733, 2001.

[14] E. Wolf, "A macroscopic theory of interference and diffraction of light from finite sources. I. Fields with a narrow spectral range," Proceedings of the Royal Society of London. Series A, vol. 225, pp. 96-111, 1954.

[15] E. Wolf, "A macroscopic theory of interference and diffraction of light from finite sources. II. Fields with a spectral range of arbitrary width," Proceedings of the Royal Society of London. Series A, vol. 230, pp. 246-265, 1955.

[16] A. Papoulis, Systems and Transforms with Applications in Optics, McGraw-Hill, New York, NY, USA, 1968.

[17] M. J. Bastiaans, "A frequency-domain treatment of partial coherence," Optica Acta, vol. 24, no. 3, pp. 261-274, 1977.

[18] L. Mandel and E. Wolf, "Spectral coherence and the concept of cross-spectral purity," Journal of the Optical Society of America, vol. 66, no. 6, pp. 529-535, 1976.

[19] A. Walther, "Radiometry and coherence," Journal of the Optical Society of America, vol. 58, no. 9, pp. 1256-1259, 1968.

[20] A. Walther, "Propagation of the generalized radiance through lenses," Journal of the Optical Society of America, vol. 68, no. 11, pp. 1606-1610, 1978.

[21] D. Dragoman, "Applications of the Wigner distribution function in signal processing," EURASIP Journal on Applied Signal Processing, vol. 2005, no. 10, pp. 1520-1534, 2005.

[22] K. B. Wolf, Integral Transforms in Science and Engineering, chapter 9, Plenum Press, New York, NY, USA, 1979.

[23] A. W. Lohmann, "Image rotation, Wigner rotation, and the fractional Fourier transform," Journal of the Optical Society of America $\{A\}$, vol. 10, no. 10, pp. 2181-2186, 1993.

[24] M. J. Bastiaans and T. Alieva, "Wigner distribution moments measured as fractional Fourier transform intensity moments," in Proc. 19th Congress of the International Commission for Optics, Optics for the Quality of Life (ICO-19), A. Consortini and G. C. Righini, Eds., vol. 4829 of Proceedings of SPIE, pp. 245 246, Firenze, Italy, August 2002.

[25] M. J. Bastiaans and T. Alieva, "Wigner distribution moments in fractional Fourier transform systems," Journal of the Optical Society of America $\{A\}$, vol. 19, no. 9, pp. 1763-1773, 2002.

Martin J. Bastiaans was born in Helmond, The Netherlands, in 1947. He received the M.S. degree in electrical engineering (with honours) and the Ph.D. degree in technical sciences from the Technische Universiteit Eindhoven (Eindhoven University of Technology), Eindhoven, The Netherlands, in 1969 and 1983, respectively. In 1969 he became an Assistant Professor and since 1985 he has been an Associate Professor with the Department of Electrical Engineering, Technische Universiteit Eindhoven, in the Signal Processing Systems Group, where he teaches electrical circuit analysis, signal theory, digital signal processing, and Fourier optics and holography. His main current research interest is in describing signals by means of a local frequency spectrum (for instance, the Wigner distribution function, the windowed Fourier transform, Gabor's signal expansion, etc.). He is the author and coauthor of more than 140 papers in international scientific journals and proceedings of scientific conferences. Dr. Bastiaans is a Fellow of the Optical Society of America and a Senior Member of the Institute of Electrical and Electronics Engineers.
Tatiana Alieva was born in Moscow, Russia. In 1983 and 1996 she received the M.S. and Ph.D. degrees (with honours) in physics from M. V. Lomonosov Moscow State University and University Autonoma of Madrid, respectively. From 1983 till 1999, she worked for A. L. Mintz Radiotechnical Institute, Academy of Science, Moscow. She spent several years as a Postdoctoral Fellow at the Departments of Electrical En-

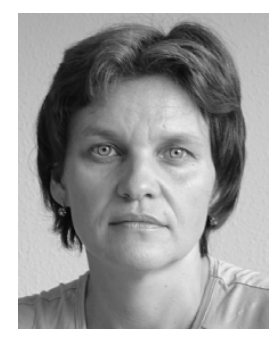
gineering of Catholic University of Leuven, Belgium, and Eindhoven University of Technology, The Netherlands. She is currently a Researcher at the Physical Faculty of Complutense University of Madrid. She has worked in diverse fields of physics, such as theory of high energetic beams channelling in crystals, and radio waves scattering on the anisotropic formations in the ionosphere. Her current research interests are in optical information processing, fractal signal analysis, theory of fractional integral transforms, phase retrieval, and characterization of optical fields by means of phase-space distributions. She is an author of more than eighty scientific publications. 